Abstract

\title{
Cytotoxic and Antiinflammatory Activity Guided Studies on Plantago holosteum Scop. ${ }^{\dagger}$
}

\author{
Yasin Genc *, U. Sebnem Harput and Iclal Saracoglu \\ Department of Pharmacognosy, Faculty of Pharmacy, Hacettepe University, Sihhiye, Ankara 06100, Turkey; \\ isaracog@gmail.com (I.S.) \\ * Correspondence: ygncyasin@gmail.com; Tel.: +90-312-305-1089 \\ + Presented at the 2nd International Conference on Natural Products for Cancer Prevention and Therapy, \\ Kayseri, Turkey, 8-11 November 2017.
}

Published: 14 November 2017

\begin{abstract}
The genus Plantago (Plantaginaceae) is represented by 21 species in Turkey. Plantago species are used as folk medicine, especially for wound healing, antiinflammatory and anticancer effects. Iridoid glucosides, phenylethanoid and flavonoid glycosides, caffeic acid derivatives, polysaccharides and lipids are main components of Plantago species. In this study; aerial parts of Plantago holosteum Scop. were investigated by bioactivity guided fractionation via cytotoxic and antiinflammatory effects. For this purpose; aqueous extract of plant, fractions and compounds isolated from active fractions were tested for their cytotoxic effects against HEp-2 cancer, L929 and RAW 264.7 noncancerous cell lines using MTT assay while anti-inflammatory effects were monitorized by the level of nitric oxide (NO) on LPS stimulated RAW264.7 macrophages. Furthermore; DPPH, NO, SO and ABTS radical scavenging capacities were also investigated to support this study. According to our results; aqueous extract and fractions showed selective cytotoxic effect on HEp-2 cancer cells and reduced the NO level with their increasing concentrations. Moreover, acteoside and isoacteoside isolated from active fractions, showed selective cytotoxic activity against HEp-2 cells and decreased the level of NO not compatible with increasing concentrations; thus cytotoxic and anti-inflammatory activities of aqueous extract of $P$. holosteum can be attributed to these compounds.
\end{abstract}

Keywords: Plantago species; cytotoxic effect; anti-inflammatory effect

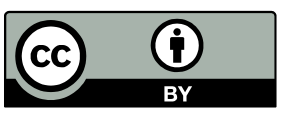

(C) 2017 by the authors. Licensee MDPI, Basel, Switzerland. This article is an open access article distributed under the terms and conditions of the Creative Commons Attribution (CC BY) license (http://creativecommons.org/licenses/by/4.0/). 